Published in final edited form as:

Int J Inj Contr Saf Promot. 2010 June ; 17(2): 79-85. doi:10.1080/17457301003786948.

\title{
Sustainable improvements in injury surveillance in Ghana
}

\author{
Koranteng Adofo ${ }^{1}$, Peter Donkor ${ }^{1}$, Francis Afukaar ${ }^{2}$, Kofi Adomako Boateng ${ }^{1}$, and Charles \\ Mock $^{3}$ \\ ${ }^{1}$ Kwame Nkrumah University of Science and Technology and Komfo Anokye Teaching Hospital \\ ${ }^{2}$ Building and Roads Research Institute, University P.O. Box UPO 40, Kumasi, Ghana \\ ${ }^{3}$ Harborview Injury Prevention and Research Center, University of Washington, Seattle, USA
}

\section{Abstract}

Introduction-The mortuary is an important foundation for injury surveillance. However, mortuary data are incomplete in many developing countries.

Methods-The KATH mortuary handles most injury deaths for Kumasi, Ghana. During 1994-5, many cases in KATH's mortuary logbooks had missing information deaths.

A low-cost pilot program was adopted to improve recording of injury deaths. During 1996-9, 633 deaths/year were recorded.

Results-Project sustainability assessment in 2006 showed that reporting was high, with 773 cases per year. Data quality was standard with similar percents of missing values for key variables compared with the pilot period. Supplemental data constituting $20 \%$ was obtained from the ICU, for which data recording in the mortuary was incomplete.

Conclusion-Low-cost improvements can lead to improved mortuary reporting of injury deaths. Collation of data from multiple sources remains a problem at KATH. Improved organization and training could remedy the situation.

\section{Keywords}

injury; surveillance; developing country; low- and middle-income country

\section{Introduction}

Injury has become a huge health problem globally, especially in low- and middle-income countries (LMICs). In order to address this problem, accurate information is needed on the

Corresponding Author: Koranteng Adofo, Department of Surgery, SMS /KNUST, University Post Office (PMB) Kumasi, Ghana. Tel/ Fax: + 2335122307 , adofokoranteng@yahoo.com.

Co-Authors

Peter Donkor (KNUST), Department of Surgery, SMS /KNUST, University Post Office (PMB) Kumasi, Ghana. Tel/Fax: + 23351 40819, petadonkor@yahoo.com

Francis Afukaar (BRRI / CSIR, Ghana), Building and Roads Research Institute, University P.O. Box UPO 40, Kumasi, Ghana. Tel: +233 51 60080; Fax: +233 271771602; fkafukaar@yahoo.com

Kofi Adomako Boateng (KNUST), Department of Pathology, SMS /KNUST, University Post Office (PMB) Kumasi, Ghana. Tel/Fax: +2335124616, catcap05@yahoo.com

Charles Mock (HIPRC), Harborview Injury Prevention and Research Center (HIPRC), Box 359960, Harborview Medical Center, 325

Ninth Avenue, Seattle, WA 98104, USA, Tel: +1-206-744-9430, cmock@u.washington.edu 
extent and nature of injury and injury-related deaths. The usual sources of such information are: vital statistics (e.g. death certificates or records from mortuaries), police reports of vehicle crashes or other injury producing events, and health service records. All of these sources have been shown to have shortcomings, especially under-reporting, in many LMICs (Mock et al. 2004).

Many factors lead to such under-reporting, including limited resources. However, it is possible and necessary to make low-cost and sustainable improvements in injury surveillance in LMICs, improvements that will enable advances in all other aspects of injury control (Ghaffar et al. 1999; Kobusingye et al. 2000; Rahman et al. 2000; Holder et al. 2001).

In Ghana, there have been recent efforts to strengthen the collection of injury data from several main sources. The goal of the current study is to assess the effects of these efforts as regards mortuary data. In particular, one of the main sources of data on fatally injured persons in Ghana's second largest city, Kumasi, is the mortuary at the Komfo Anokye Teaching Hospital (KATH). Prior to 1996, many cases in KATH's mortuary logbooks had missing or vague information on cause of death, and only 70 injury deaths/year were reported. This was felt to be a gross under-estimate of injury among Kumasi's more than one million people. A pilot program hired several part-time nurses (one full-time-equivalent) to improve recording of injury deaths. These nurses visited the mortuary daily to review the logbook and discuss the causes of death with mortuary staff, including doctors, recordkeepers, and laborers, who were often aware of the mechanisms of injury through contact with the victims' families. Missing information was then entered into the mortuary logbook. Supplemental information was obtained from the logbooks on various wards and from medical records. Likewise, these ward logbooks were used to identify patients who had died from injuries but who were not recorded in the mortuary logbooks. As a result, during this project (1996-9), reporting of injury deaths in KATH's mortuary increased to 633 deaths/ year, a much more accurate reflection of injury's toll in Kumasi (London et al. 2002).

In the current study, we wished to evaluate how sustainable these improvements were after the funding for the pilot project expired and the additional staff time was not being specifically devoted to injury data recording. A secondary goal of the study was to provide basic descriptive epidemiology of injury in the study area to help guide other aspects of injury control, including prevention and care.

\section{Methods}

This study was conducted in Kumasi (population 1,400,000), at KATH, which has 1000 beds and is the city's main hospital. KATH's mortuary is a holding area for the bodies of patients who die in the hospital and for those who are dead on arrival. It is also used to store the bodies of some persons who die outside the hospital until funeral arrangements are made. Although the mortuary is mainly used for the bodies of person who have resided and died in Kumasi, it is also variably used by surrounding communities. The mortuary is administered by the Pathology Department, which provides a range of services to the hospital, including autopsies and histology. 
The logbook of the KATH mortuary contains name, age, date and site of death, and cause of death for all persons whose remains are kept at the mortuary. This includes people who die at or upon presentation to the hospital, as well as people who die outside the hospital and whose bodies are brought for storage at the mortuary. The logbook of the mortuary was reviewed for all injury-related deaths recorded for the years 2005-2006. Injury-related deaths were discerned by looking for causes of death reflecting either injury-related mechanisms (e.g. motor vehicle crash) or specific anatomic injuries listed as cause of death (e.g. head injury).

For persons who died in hospital, supplemental information was obtained from the logbooks of the various wards. We sought to identify persons who had died from injury who were not recorded at the mortuary. In hospital, injured patients are initially assessed in the casualty ward, where resources are limited. If urgent resuscitation is required, patients are triaged to the intensive care unit (ICU) which is adjacent to the casualty ward. Patients who are stable are sent from either of these locations to the main hospital wards. All of these locations maintain their own logbooks. Likewise, many fatally injured persons have autopsies performed, data about which are kept in a separate logbook.

Data from all of these sources were collected on a one-page data collection form, which addressed basic demographics, mechanism of injury, location of injury producing event, and site of death. Data were abstracted and collected by one of the authors (KA), assisted by two research assistants who were personnel from the medical records department. Quality assurance was provided by feedback from senior staff who served as on-site supervisors $(\mathrm{PD}, \mathrm{KB})$. Data gathering and quality assurance were aided by the fact that the on-site supervisors and data abstracters all worked at KATH, either in data management, the mortuary, or in patient care. Data were coded, entered, and analyzed using EpiData 3.0 and SPSS 15.0. Analysis focused on the main goal of the study, examining items that would constitute a successful continuation of the pilot programme, including continuation of an overall high number of injury cases and continued high data quality as reflected by percent missing for key variables. Analysis also focused on the secondary goal of the study, providing basic descriptive epidemiologic data to assist with other aspects of injury control. This study was approved by the Kwame Nkrumah University of Science and Technology IRB and granted an exemption from review by the University of Washington IRB.

\section{Results}

\subsection{Demographic data}

During the two-year study period, 1545 injury-related deaths were recorded (680 in 2005, 865 in 2006), or an average of 773 deaths per year. Of these deaths, $73 \%$ were males and $27 \%$ females. Mean age was $32.7 \pm 19.0$ (SD) years. Injury-related deaths accounted for $9.9 \%$ of all 15,467 deaths recorded in the mortuary during the study period.

\subsection{Sustainability of prior improvements in reporting and data quality}

An assessment of the sustainability of the mortuary data improvements can be obtained by comparing the overall reporting and the data quality from the current study with that 
reported during 1996-9 when two part-time research assistants were working to assure accurate injury data collection.

In terms of overall reporting, the number of cases reported each year remained high at 773 per year, compared with 633 during the pilot project period of 1996-9. It is difficult to know if the higher numbers for 2005-6 reflect improved data capture, increased number of cases due to a higher population, or increased injury rate. However, it can certainly be seen that the rate of capture stayed much higher than the rate of only 70 cases/year being reported before 1996. There were two new mechanisms of injury added to the current study compared with the 1990's data: drowning and poisoning. Excluding these, the reporting for 2005-6 is at 723 per year, still higher than the 1996-9 period.

Data quality can be assessed, in part, by looking at the percent of variables listed as missing or unknown. As shown in Table 1, the percent of such missing/unknown was higher for some variables in 1996-9 and higher for others during 2005-6. For 2005-6, there had been some improvements in percentage of recording of age, gender, and place of death. However, there had been some deterioration in recording of place of injury producing event, with unknown/not recorded rising from $2 \%$ in $1996-9$ to $13 \%$ in $2005-6$. Thus, overall, data quality had been maintained compared with the pilot project.

As in the pilot project, there was a need to obtain supplemental data on deaths that occurred elsewhere in the hospital. In particular, mortuary data had been poorly recorded for many of the 301 ICU deaths. Twenty percent of these had not even been recorded in the mortuary log book and 35\% had been recorded but with such vague information that it could not have been ascertained that they were injury deaths. Most of the remainder still required acquisition of supplemental data from the ICU logs to be able to complete data collection.

\subsection{Mechanism of injury}

Table 2 shows the mechanisms of injury according to age. Transport-related injuries accounted for a total of $61 \%$ of injury-related deaths, with $49 \%$ of these being listed as motor vehicle crashes and $12 \%$ being listed as pedestrian injuries. Transport-related injuries (both motor vehicle crashes and pedestrian injuries) were the leading cause of injury death among all age groups. The second leading cause was violence, accounting for $10 \%$ of injury deaths. Burns and drowning were significant causes among children aged $0-4$ years. Suicides were nearly equally divided between poisoning $(n=46)$ and hanging $(n=42)$.

The distribution of mechanism of injury differed by gender for two mechanisms. Burns were more likely to occur among females (6\% of all female injuries) compared with males (3\%). Violence was more likely to occur among males (12\% of all male injuries were violence related) compared with females $(6 \%)$. Other mechanisms were approximately equally distributed by gender.

The majority of injury producing events (61\%) occurred on roadways (Table 3), in light of the leading mechanisms being road traffic related. The next largest group of injuries occurred in homes (13\%) and workplaces (6\%). Location had not been recorded for $13 \%$. 


\subsection{Site of death}

The majority (76\%) of fatally injured persons were brought in dead to the hospital (Table 4). This designation does not distinguish between those who were brought in to the casualty ward and were dead on arrival versus those who were clearly dead in the field and brought directly to the mortuary. The next most common site of death was the ICU (19.5\%), with very small numbers dying at other locations in the hospital, including the main hospital wards $(3.9 \%)$ and casualty ward $(0.4 \%)$.

\subsection{Comparison between two periods}

Table 5 summarizes the differences between the two study periods. There were differences in data gathering and coding between the periods. Drowning and poisoning were not assessed and electrocutions were bundled with burns in the first period. Other transport in the first period included some smaller mechanisms such as motorcycle crashes and falling from moving vehicles, which were bundled with motor vehicle crashes in the second period. Despite these differences, the increase in reporting of suicides and violence is notable.

\section{Discussion}

Efforts to decrease the toll from injuries rely on accurate and timely data on the incidence and nature of the injuries sustained. Such information is needed to ascertain which injury control efforts have been successful, and thus should be continued or expanded, and which ones have failed, and thus should be amended or discontinued. Surveillance systems to provide this important information are inadequate in most LMICs, with significant underreporting and with shortcomings in the data quality. There is growing worldwide attention to find low-cost and sustainable methods to strengthen data sources for injury control (Holder et al. 2001). This study sought to evaluate the sustainability of a programme to improve the reliability and accuracy of injury data in the main mortuary in an African city.

Before drawing conclusions from the results, the limitations of the study methods must be addressed. The main limitation was that there is no gold standard against which to compare the mortuary data. There had been a household survey on injury conducted in Kumasi in 1995-6, which showed annual rates of overall injury mortality of 83/100,000 and of transport-related mortality of 67/100,000 (Mock et al. 1999). However, confidence intervals for the mortality estimates were wide and a similar household survey has not been reported since then. Given this lack of a gold standard for comparison, it is reasonable to compare overall numbers of cases recorded against prior periods when there had been no improvements (before 1996) and to a period when resources were being devoted to improved injury data collection (1996-9). Likewise, the percent of variables coded as unknown or missing is a reasonable reflection of data quality.

Despite these limitations, this study allows meaningful conclusions to be drawn about the sustainability of programs to improve injury data recording at mortuaries in LMICs. The major finding is that recording of injury deaths remained high after the funding ended. The overall number of injury deaths recorded went slightly up in 2005-6 compared to 1996-9. Without a gold standard for comparison, it cannot be ascertained whether the percentage of capture went slightly up or down. However, the numbers recorded during both periods 
(1996-9 and 2005-6) remain dramatically higher than the 70 injury-related deaths reported per year before 1996 and thus likely represent a more accurate reflection of the real picture of injury mortality in the city. Whatever changes may have occurred in data capture between 1996-9 and 2005-6 are small in comparison to the sustained improvement that occurred with the investments made in 1996.

Likewise, in terms of data quality, the two periods during the mortuary study both showed moderate levels of unknown/missing coding for several key variables. One other variable that may reflect the data quality is that of mechanism of injury. In 1996-9, 50\% of all injury deaths were pedestrian, which was higher than the $34 \%$ of all injury deaths from motor vehicle crashes. In 2005-6, these figures had changed to only $12 \%$ for pedestrian injuries and to $49 \%$ for motor vehicle related crashes. It is possible that the rate of pedestrian injuries has indeed gone down. However, it seems unlikely to have gone down so dramatically. Moreover, ancillary data from police reports shows that pedestrians accounted for $40 \%$ of police-reported road traffic injuries (Afukaar et al. 2006). Thus, it is likely that at least in part the decrease in pedestrian injuries reflects a decrease in data quality, with some pedestrian injuries being recorded in the more general category of motor vehicle related injury. On the other hand, $6 \%$ of injury deaths in 2005-6 were coded as suicide, a mechanism scarcely reported in 1996-9, indicating likely improvement in recording for that mechanism.

The data from this study have implications for several injury control activities: prevention, care, and surveillance. In terms of prevention, the study emphasizes the continued need for attention to road safety in this environment, as elsewhere globally (Peden et al. 2004). It also demonstrates the importance of burn prevention and drowning prevention efforts for young children (Peden et al. 2008). Finally, the study has shown a tragic increase in violencerelated deaths, which increased from 16 per year in 1996-9 to 77 per year in 2005-6. Thus, in addition to road safety, violence prevention stands out as a growing priority in the study area (Krug et al. 2002).

In terms of trauma care, the study shows a preponderance (76\%) of prehospital deaths. This is minimally changed from the $80 \%$ reported in 1996-9. This emphasizes the importance of efforts to improve prehospital care. Although improvements in hospital-based care are certainly important, the fact that the vast majority of injured people continue to die in the field, indicates that efforts to improve prehospital care are more likely to affect overall injury mortality.

The major implications of this study relate to methods for improving injury surveillance in LMICs. Most importantly, this study has shown that a small investment in improving data capture and quality can be sustainable over the long-term with no additional funding. It is difficult to know exactly what factors at KATH led to the successful continuation of the pilot programme when many pilot programmes fail after funding ends. In part, this may have been due to a growth in the number of staff working in the mortuary (10 in 1996 to 20 in 2006) and in the number of pathologists at KATH (1 in 1996 to 4 in 2006). In part however, the improvement is also due to changes in administrative procedures and training. The 1996-9 study revealed weaknesses in the system and prompted changes to improve data 
management in the mortuary. This included better on-the-job training for mortuary attendants on accurate recording of information, as well as infection control and general mortuary practice. Likewise, there has been a change in procedure whereby information on cases is recorded at entrance, rather than afterwards.

Similar improvements should be eminently achievable in countries worldwide and a few reports of similar improvements have been published. For example, in South Africa, the National Non-Natural Mortality Surveillance System is built upon a nationwide network of mortuaries. The reporting of injury deaths from these mortuaries has been strengthened by investments in training of staff (e.g. mortuary administrative personnel) and by use of a simple, standardized case report form. This low-cost system has been found to provide timely and useful information for injury control efforts (Butchart et al. 2001).

In Maputo, Mozambique, low-cost efforts to strengthen the overall mortality recording system included combining data from several civil registry offices into one register, along with improvements in coding of deaths using the International Classification of Diseases-9 and supplementing the data with autopsy records, when available. This system provided better information on burden of disease in general and better surveillance data for road safety (Dgedge et al. 2001; Romao et al. 2003; Nizamo et al. 2006).

In Colombia, observatories have been implemented in many municipalities. These incorporate data from death certificates and multiple other sources to give a low-cost, rapidly available picture of injury mortality. These have allowed cities to develop more responsive policies and injury prevention programmes (Gutierrez-Martinez et al. 2007).

The current study has likewise identified several weaknesses in the mortuary recording system that need to be addressed. First, a significant number of cases were not well recorded in the mortuary logbooks, and required supplemental data gathering from other hospital sources, especially the ICU. Use of data for injury surveillance could be facilitated and made more timely by collation of these separate data sources that are already within the same institution. One of the measures to achieve this, computerization of records, would require a modest resource investment. Such computerization would likely reduce the data loss that often goes with the present manual documentation method. Computerization would also facilitate efficient data storage, retrieval, analysis, and dissemination of the data. Although the level of reporting in the current system is noteworthy, it still requires considerable time and effort in reviewing the logbooks to extract data for surveillance purposes. One must also consider the need for improvements in the data quality in the original death certificates.

An additional measure that would be very inexpensive would be assignment of a unique institutional identification number. Currently, the various locations in the hospital, including the mortuary, utilize their own numbering systems. A unique identification number that would be operational in all hospital departments would assist collation of data from multiple sources (Holder et al. 2001).

The modest frequency of unknown/missing variables and overall data quality could be improved upon by the dual methods of improving staff training and by standardization of the data gathered in the mortuary. For example, WHO has published Guidelines for Injury 
Surveillance (Holder et al. 2001) that contains a "form to collect core minimum data on any case of injury." Utilizing this for all injury cases in the mortuary would be a method for improving data quality. Of course, an additional data gathering form would need to be harmonized with existing mortuary procedures, so that minimal additional work is created for the staff. Otherwise, the sustainability of the suggested data gathering improvements would be jeopardized.

\section{Conclusions}

The mortuary is an important foundation for injury surveillance. However, mortuary data are incomplete in many developing countries. Low-cost improvements can lead to improved reporting and data quality. This study has shown that such improvements are sustainable after the initial investment ends. Some problems remain, including insufficient collation of data from multiple sources in the same institution and use of general, rather than specific, coding of mechanism. These smaller problems could be corrected by improved organization and training, as well as by small investments such as computerization of records, use of a unique identification number that would be the same in all hospital departments, and standardization of data gathering at time of mortuary registration.

\section{Acknowledgments}

This study was funded, in part, by grant (D43-TW007267) from Fogarty International Center, US NIH and by grant (R49-CE000197) from US CDC. The authors thank the staff of the KATH mortuary for their advice and assistance with data gathering and with understanding the mortuary system and ways to strengthen it.

\section{References}

Afukaar, FK.; Agyemang, W.; Mosi, I. Building and Road Research Institute Report on Road Traffic Accidents in Ghana. Kumasi: Building and Roads Research Institute; 2006.

Butchart A, Peden M, Matzopoulos R, Phillips R, Burrows S, Bhagwandin N, Saayman G, Cooper A. The South African National Non-Natural Mortality Surveillance System - rationale, pilot results and evaluation. S Afr Med J. 2001; 91:408-417. [PubMed: 11455806]

Dgedge M, Novoa A, Macassa G, Sacarlal J, Black J, Michaud C, Cliff J. The burden of disease in Maputo City, Mozambique: registered and autopsied deaths in 1994. Bull WHO. 2001; 79:546-552. [PubMed: 11436477]

Ghaffar A, Hyder AA, Mastoor MI, Shaikh I. Injuries in Pakistan: directions for future health policy. Health Policy and Planning. 1999; 14(1):11-17. [PubMed: 10351465]

Gutierrez-Martinez M, et al. The evaluation of a surveillance system for violence and non-intentional injury mortality in Colombia cities. Inj Control Saf Promot. 2007; 14:77-84.

Holder, Y.; Peden, M.; Krug, E.; Lund, J.; Gururaj, G.; Koibusingye, O. Injury Surveillance Guidelines. Geneva: World Health Organization; 2001.

Kobusingye, Lett RR. Hospital based trauma registries in Uganda. J Trauma. 2000; 48:498-502. [PubMed: 10744292]

Krug, E.; Dahlberg, L.; Mercy, J.; Zwi, A.; Lozano, R. World Report on Violence and Health. Geneva: World Health Organization; 2002.

London J, Mock CN, Abantanga FA, Quansah RE, Boateng KA. Using mortuary statistics in the development of an injury surveillance system in Ghana. Bull WHO. 2002; 80:357-364. [PubMed: 12077610]

Mock C, Quansah R, Krishnan R, Arreola-Risa C, Rivara F. Strengthening the prevention and care of injuries worldwide. Lancet. 2004; 363:2172-2179. [PubMed: 15220042] 
Mock CN, Abantanga F, Cummings P, Koepsell TD. Incidence and outcome of injury in Ghana: results of a community-based survey. Bulletin of the World Health Organization. 1999; 77:955964. [PubMed: 10680242]

Nizamo H, Meyrowitsch D, Zacarias E, Konradsen F. Mortality due to injuries in Maputo City, Mozambique. Int J Injury Contr Saf Promot. 2006; 13:1-6.

Peden, M.; Oyegbite, K.; Ozanne-Smith, J.; Hyder, A.; Branche, C.; Rahman, A.; Rivara, F.; Bartolomeos, K. World report on child injury prevention. Geneva: World Health Organization; 2008.

Peden, M.; Scurfield, R.; Sleet, D.; Mohan, D.; Hyder, A.; Jarawan, E.; Mathers, C. World Report on Road Traffic Injury Prevention. Geneva: World Health Organization; 2004.

Rahman F, Andersson R, Svanstrom L. Potential of using existing injury information for injury surveillance at the local level in developing countries. Public Health. 2000; 114:133-136. [PubMed: 10800153]

Romao F, Nizamo H, Mapasse D, Rafico M, Jose J, Mataruca S, Efron M, Omondi L, Leifert T, Bicho J. Road traffic injuries in Mozambique. Inj Control Saf Promot. 2003; 10:63-67. [PubMed: 12772487] 


\section{Table 1}

Changes in data quality as reflected by percentage of unknown/missing data for 1996-9 (London et al. 2002) compared with 2005-6.

\begin{tabular}{lrr}
\hline Variable & 1996-9 & $\mathbf{2 0 0 5 - 6}$ \\
\hline Age & $9 \%$ & $2 \%$ \\
Gender & $2 \%$ & $0 \%$ \\
Mechanism of injury & $5 \%$ & $6 \%$ \\
Place of injury producing event & $2 \%$ & $13 \%$ \\
Place of death & $2 \%$ & $0 \%$ \\
\hline
\end{tabular}




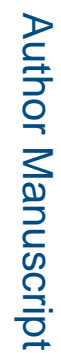

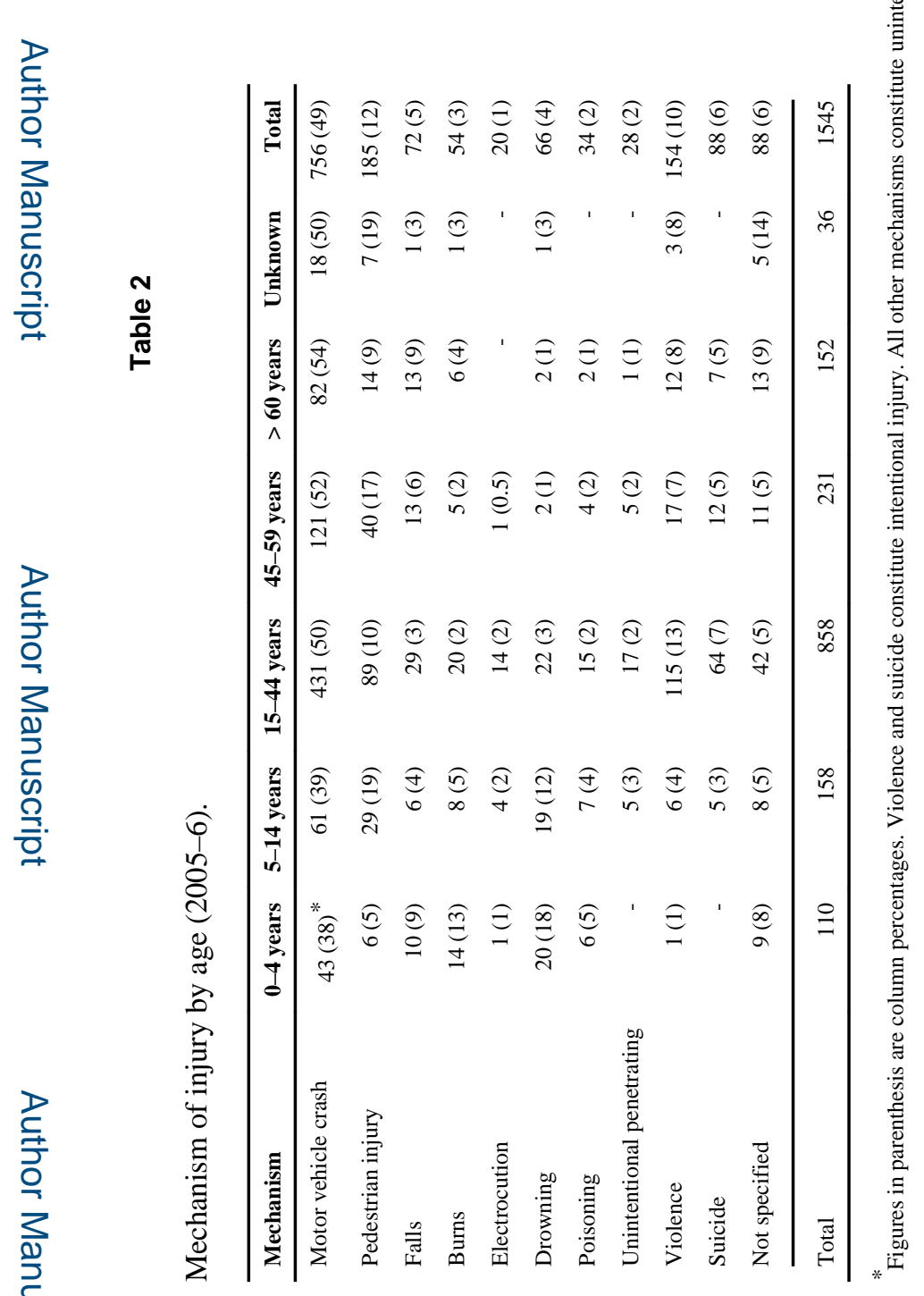




\section{Table 3}

Location of injury producing events (2005-6).

\begin{tabular}{lr}
\hline Location & No. of persons \\
\hline Highway / Street & $935(61)^{*}$ \\
Home & $195(13)$ \\
School & $1(0.1)$ \\
Work Place & $88(5.7)$ \\
Farm / Garden / Bush & $71(4.6)$ \\
Water body & $55(3.6)$ \\
Unspecified & $200(13)$ \\
\hline Total & 1545 \\
\hline
\end{tabular}

Figures in parenthesis are percentages. 


\section{Table 4}

Place of death (2005-6).

\begin{tabular}{lr}
\hline Place of death & No. of persons \\
\hline Brought in dead & $1177(76)^{*}$ \\
Casualty & $6(0.4)$ \\
Intensive care unit & $301(19.5)$ \\
Ward & $61(3.9)$ \\
\hline Total & 1545 \\
\hline
\end{tabular}

* Figures in parenthesis are percentages.

Brought in dead implies prehospital deaths. 
Table 5

Changes in reporting of mechanisms of injury for 1996-9 (London et al. 2002) compared with 2005-6.

\begin{tabular}{lrr}
\hline Mechanism & 1996-1999 $^{\mathbf{1}}$ & $\mathbf{2 0 0 5 - 2 0 0 6}$ \\
\hline Motor vehicle crash & $650(34)^{*}$ & $756(49)$ \\
Pedestrian injury & $953(50)$ & $185(12)$ \\
Other transport & $74(4)$ & 0 \\
Falls & $30(2)$ & $72(5)$ \\
Burns & $28(2)$ & $54(3)$ \\
Electrocution & 0 & $20(1)$ \\
Drowning & Not assessed & $66(4)$ \\
Poisoning & Not assessed & $34(2)$ \\
Unintentional penetrating wounds & $20(1)$ & $28(2)$ \\
Violence & $47(3)$ & $154(10)$ \\
Suicide & 0 & $88(6)$ \\
Not specified & $96(5)$ & $88(6)$ \\
\hline Total & $1898(100)$ & $1545(100)$ \\
\hline
\end{tabular}

Figures in parenthesis are percentages. 